\title{
Early and consistent overexpression of ADRM1 in ovarian high-grade serous carcinoma
}

\author{
Rosie T. Jiang ${ }^{1}$, Anna Yemelyanova ${ }^{1}$, Deyin Xing ${ }^{1}$, Ravi K. Anchoori ${ }^{2}$, Jun Hamazaki ${ }^{5}$, Shigeo Murata ${ }^{5}$,
} Jeffrey D. Seidman ${ }^{6}$, Tian-Li Wang ${ }^{4}$ and Richard B. S. Roden ${ }^{1,2,3^{*}}$ (D)

\begin{abstract}
Background: Ovarian carcinoma is highly dependent on the ubiquitin proteasome system (UPS), but its clinical response to treatment with the proteasome inhibitor bortezomib has been disappointing. This has driven exploration of alternate approaches to target the UPS in ovarian cancer. Recently, proteasome inhibitors targeting the 195 regulatory particle-associated RPN13 protein have been described, such as RA190. RPN13, which is encoded by ADRM1, facilitates the recognition by the proteasome of its polyubiquinated substrates. Inhibition of RPN13 produces a rapid, toxic accumulation of polyubiquitinated proteins in ovarian and other cancer cells, triggering apoptosis.

Here, we sought to determine if RPN13 is available as a target in precursors of ovarian/fallopian tube cancer as well as all advanced cases, and the impact of increased ADRM1 gene copy number on sensitivity of ovarian cancer to RA190.
\end{abstract}

Methods: ADRM1 mRNA was quantified by RNAscope in situ hybridization and RPN13 protein detected by immunohistochemistry in high grade serous carcinoma (HGSC) of the ovary and serous tubal intraepithelial carcinoma (STIC). Amplification of ADRM1 and sensitivity to RA190 were determined in ovarian cancer cell lines.

Results: Here, we demonstrate that expression of ADRM1mRNA is significantly elevated in STIC and HGSC as compared to normal fallopian tube epithelium. ADRM1 mRNA and RPN13 were ubiquitously and robustly expressed in ovarian carcinoma tissue and cell lines. No correlation was found between ADRM1 amplification and sensitivity of ovarian cancer cell lines to RA190, but all were susceptible.

Conclusions: RPN13 can potentially be targeted by RA190 in both in situ and metastatic ovarian carcinoma. Ovarian cancer cell lines are sensitive to RA190 regardless of whether the ADRM1 gene is amplified.

Keywords: ADRM1, RPN13, STIC, Proteasome inhibitor, Ovarian/fallopian tube cancer

\section{Background}

Missense mutation of TP53 is the dominant driver in ovarian/fallopian tube cancer [1-4]. Indeed TP53 is mutated in nearly all high grade serous carcinomas (HGSC), the histotype responsible for most deaths from ovarian cancer $[5,6]$, and is also mutated in its precursor, serous tubal intraepithelial carcinoma (STIC) [7-9], suggesting

\footnotetext{
* Correspondence: roden@jhmi.edu

'Department of Pathology, The Johns Hopkins University, Baltimore, MD 21231, USA

2Department of Oncology, The Johns Hopkins University, Baltimore, MD 21231, USA

Full list of author information is available at the end of the article
}

this is a key and early event in carcinogenesis [10]. In addition to inactivating wild type TP53 function, these mutations frequently confer gain-of -function properties including redirection of Nrf2 to upregulate the proteasome [11]. The aberrant metabolism of ovarian cancer cells produces an excess of misfolded proteins that are polyubiquitinated for targeted degradation via the proteasome, and this is associated with proteasome upregulation [12]. Consequently, ovarian cancer cells are both highly dependent on proteasome function and especially sensitive to treatment with a proteasome inhibitor [12-14]. The FDA-approved drug bortezomib, approved for the 
treatment of multiple myeloma and mantle cell lymphoma, inhibits 20S core particle (CP) proteolytic function producing a rapid and toxic buildup of denatured protein aggregates [15]. The cells attempt to ameliorate this stress via the unfolded protein response (UPR) which promotes re-folding via chaperone upregulation as well as the rapid sequestration and degradation of the misfolded, ubiqutinated proteins. Inability to relieve proteotoxic stress and achieve homeostasis induces TP53independent cell death [16]. Indeed, treatment with bortezomib slowed the growth of ES-2 ovarian carcinoma xenograft, although alone it did not cure the mice [12].

Despite this promise, early studies with bortezomib in patients with ovarian cancer or other solid tumors have demonstrated minimal clinical benefit. Initial phase I studies suggested a dose of $1.3 \mathrm{mg} / \mathrm{m}^{2}$ in combination with cisplatin/carboplatin, paclitaxel or pegylated liposomal doxorubicin had manageable neurotoxicity [17-22], although in combination with oxaliplatin a maximum dose of $1.0 \mathrm{mg} / \mathrm{m}^{2}$ bortezomib was suggested because of neuropathy [23]. However, a phase II study of bortezomib alone a dose of $1.3 \mathrm{mg} / \mathrm{m}^{2}$ in platinum-sensitive epithelial or primary peritoneal cancer showed minimal activity as a single agent [17]. Likewise, a phase II study of bortezomib and pegylated liposomal doxorubicin showed the combination was well tolerated, but the antitumor activity was considered insufficient to warrant further investigation [21]. Thus, despite bortezomib's efficacy against multiple myeloma, its toxicity, and limited activity in solid tumors has driven development of inhibitors of the ubiquitin-proteasome system (UPS) with alternative mechanisms to treat ovarian cancer.

RPN13, which is encoded by the gene ADRM1, is a ubiquitin receptor tethered to the $19 \mathrm{~S}$ regulatory particle (RP) of the proteasome via interaction of its amino terminal Pru domain to RPN2 [24]. The Pru domain of RPN13 and RPN10 cooperate to capture K48-linked polyubiquitin chains bound to proteasome substrates. The carboxy terminal domain of RPN13 recruits and promotes the deubiquitinase activity of UCHL5/UCH37 which removes distal ubiquitin from the polyubiquitin chains linked to these substrates [25-27]. Deubiquitination of the substrate is followed by unfolding and transfer to the 20S CP for its proteasomal degradation.

$A D R M 1$ was originally identified as ADhesion Regulating Molecule-1 in metastatic tumor cells [28]. Several observations have driven interest in RPN13's role in cancer biology; 1) early studies suggested that ADRM1 transcript levels commonly upregulated in diverse tumor types [29], 2) ADRM1 was identified as a recurrent amplification target in ovarian cancer [30-32], colorectal carcinoma [33, 34], gastric cancer $[35,36], 3)$ knockdown of ADRM1 transcripts suppressed proliferation, growth in soft agar and migration of diverse cancer lines but not normal cells [31, 34-39], 4) over-expression of RPN13 via ectopic expression of $A D R M 1$ enhanced proliferation [32, 35], 5) ADRM1-encoded RPN13 is required for cell cycle progression [40]. Fejzo et al. also observed that $A D R M 1$ overexpression is significantly correlated with higher stage, shorter time to recurrence and survival [32]. RNAi knock-down of ADRM1 transcripts in OAW42 cells triggers apoptosis, as is observed upon treatment with RA190. Conversely, using ectopic over expression of ADRM1 in ES2 cells to examine its biologic function in ovarian cancer cells, they observe that ADRM1 overexpression increases cell proliferation, migration and growth in soft agar. From these observations, Fejzo et al. proposed that ADRM1 is "an oncogene and therapeutic target for ovarian cancer" [32].

Anchoori et al. recently developed a promising anticancer drug, RA190, which covalently binds to RPN13 C88 in its Pru domain [36, 41]. RA190 has promising activity against ovarian cancer, cervical cancer and multiple myeloma (MM) in mouse models by triggering an unresolved unfolded protein response (UPR) and then TP53-independent apoptosis [41]. RPN13 expression is dramatically elevated in clinical samples of MM compared to normal plasma cells [42]. Furthermore, RPN13 knockdown by ADRM1 siRNA, or RA190 treatment causes loss of MM cell viability, including ex vivo patients' samples and bortezomib-resistant lines, via caspasedependent and UPR-related apoptosis [42]. Using CRISPR/Cas9 knockout of ADRM1, we show RA190 activity is dependent upon RPN13. Finally, RA190 treatment was well tolerated, inhibited MM xenograft tumor growth and extended survival [42]. Recently, another group developed KDT11, a reversible ligand of RPN13, which they found to be selectively toxic for MM cells. KDT11 provides further validation of RPN13 as an anticancer drug target, but does not have properties conducive to in vivo use [43]. Lu et al. identified a 38 aa peptide of RPN2 that binds to RPN13 Pru domain with $12 \mathrm{nM}$ affinity. Overexpression of this peptide in 293T cells prevents RPN13 binding to the proteasome resulting in an increase in the cellular load of poly-ubiquitinated proteins [44].

These observations support RPN13 as a treatment target for ovarian cancer, but it is not clear whether it is expressed in all cases and if ADRM1 amplification impacts sensitivity to RA190. Recent findings suggest that the precursor lesion for HGSCs, STIC, arise at the fimbriated end of the fallopian tubes and shed cancer cells that implant on the surface of the ovary and peritoneum [45-49]. STIC typically exhibit high proportions of proliferating cells and missense TP53 mutations $[48,50]$, but it is not known if they also express RPN13 (and to what level) and thus whether STIC are also potential treatable by RA190. 


\section{Results}

Validation of a highly sensitive and specific Chromogenic In Situ Hybridization (CISH) assay for ADRM1 transcript levels

To develop a sensitive ADRM1 mRNA CISH assay, we used a custom hybridization probe for the RNAscope ${ }^{\circ}$ 2.0 assay from Advanced Cell Diagnostics (ACD) that is designed for detection at the single transcript level. To establish its ability to assess mRNA levels, ADRM1 levels were measured in 4 HGSC patients with matching frozen tissue samples and formalin-fixed, paraffin embedded (FFPE) tissue blocks by mRNA CISH or qRT-PCR, respectively (Additional file 1: Figure S1A, S1B). Levels of ADRM1 mRNA measured with qRT-PCR correlated with RNAscope $^{\bullet} \mathrm{DAB}$ dots (numbers of the dot-like signals) in RNAscope ${ }^{\circ}$ demonstrating semi-quantitative nature of RNAscope 2.0 assay.

\section{Elevated ADRM1 mRNA expression in matched STICs and HGSCs}

Formalin-fixed, paraffin embedded blocks of 11 ovarian cancer cases were selected by three gynecologic pathologists who independently assessed the presence of STIC and HGSC carcinoma using H\&E, ki-67, and p53 staining to probe morphology, proliferation and, - surrogate of TP53 muational status, respectively. Of 11 patient samples, 7 FFPE blocks contained matched normal fallopian tube, STIC, and carcinoma (Fig. 1, Rows 1-3). Tumor tissue samples from an additional 13 HGSC patients were arrayed on a TMA containing 5 cores per patient (Table 1 , Fig. 1, Row 4).

The presence of detectable RNA in the tissue samples was verified by using positive control peptidyl prolyl isomerase B (PPIB) probe (Additional file 2: Figure S2). Next, we utilized the ADRM1 mRNA CISH assay to probe adjacent slides from the samples of 7 ovarian cancer patients with matched STICs, normal fallopian tube epithelium, and the additional samples from 13 HGSC patients. ADRM1 mRNA was detected in both normal fallopian tube epithelium as well as STIC (Fig. 2). Expression levels were assessed semi-quantitatively by gynecologic pathologist (DX) as higher in STIC tissue as compared to normal fallopian tube epithelium in all samples $(n=7)$. ADRM1 mRNA levels in matched invasive carcinoma on same slides were considered to be a similar to those in adjacent STIC. This implies that the elevation of ADRM1 mRNA levels is an early event that does not obviously increase further as disease progresses.

To confirm the semi-qualitative analysis of the gynecologic pathologist, automated quantification was used on the 7 STIC confirmed patients by ACD SpotStudio Software, which allows for direct quantification of target RNA molecules in single cells. After defining regions of interest (ROIs) including normal fallopian tube epithelium, STIC, and invasive high grade serous carcinoma, SpotStudio defines average cell size and quantifies DAB-stained (brown) dots per cell (Additional file 3: Figure S3). Overall, levels of ADRM1 mRNA were not significantly different between STIC and HGSC $(P=0.4)$ but each was significantly elevated as compared to matched normal epithelium ( $P=0.009$ and $P=0.003$ respectively, paired T-test). STIC ( $50 \%)$ and matched HGSC ( 33\%) showed a higher proportion of cells expressing high levels $(>6)$ of RPN13 mRNA dots per cell $(\sim 23 \%)$ as compared to normal epithelium (Fig. 3, Additional file 4: Figure S4).

To determine whether RPN13 protein is expressed in these tissues, IHC was performed on adjacent serial sections with mouse monoclonal anti-RPN13 antibody (Fig. 2). As expected for a proteasome protein, RPN13 IHC showed diffuse nuclear and cytoplasmic staining. The specificity of the immunohistochemistry assay was confirmed by comparing the staining of HCT116 wild type cells and an ADRM1 CRISPR knockout HCT116 clone (Additional file 5: Figure S5). RPN13 expression was detected in STIC and HGSC as well as normal fallopian tube epithelium although there was no clear indication of change in protein level based on this semi-quantitative technique (Fig. 2).

\section{Consistent expression of RPN13 in HGSC}

All cases tested $(n=13)$, regardless of HGSC stage, maintained expression of both ADRM1 mRNA (determined by in situ hybridization) and RPN13 protein as visualized by immunohistochemistry (Fig. 4). Expression of RPN13 protein was further confirmed by Western blotting of same HGSC samples $(n=4)$ used for RNA-CISH verification (Additional file 1: Figure S1C). When grouped by similar $P P I B$ mRNA levels, tissue exhibiting variable ADRM1 mRNA levels exhibited similar amounts of RPN13 (Additional file 6: Figure S6, Additional file 7: Figure S7).

\section{Amplification of ADRM1 does not correlate with expression of RPN13 or sensitivity to RA190}

The Cancer Genome Atlas (TCGA) Research Network collected tumor specimens and analyzed key genomic changes by mRNA-seq, whole exome DNA-seq, miRNAseq, methylation, copy number, in over 11,000 patient samples encompassing 33 different types of cancer [51, 52]. From analysis of TCGA data it is apparent that ovarian cancer has a higher frequency of $A D R M 1$ amplification $(\sim 8-15 \%)$ as compared to other cancers (Fig. 5a) and amplification is moderately correlated with elevated mRNA expression level (Pearson coefficient $=0.538$, Fig. 5b). ADRM1 amplification and mRNA overexpression could lead to differences in sensitivity to RPN13 inhibitors, such as RA190. To test this hypothesis, we established the copy number variation status for a panel of $n=7$ ovarian cancer cell lines in comparison to the SV40 


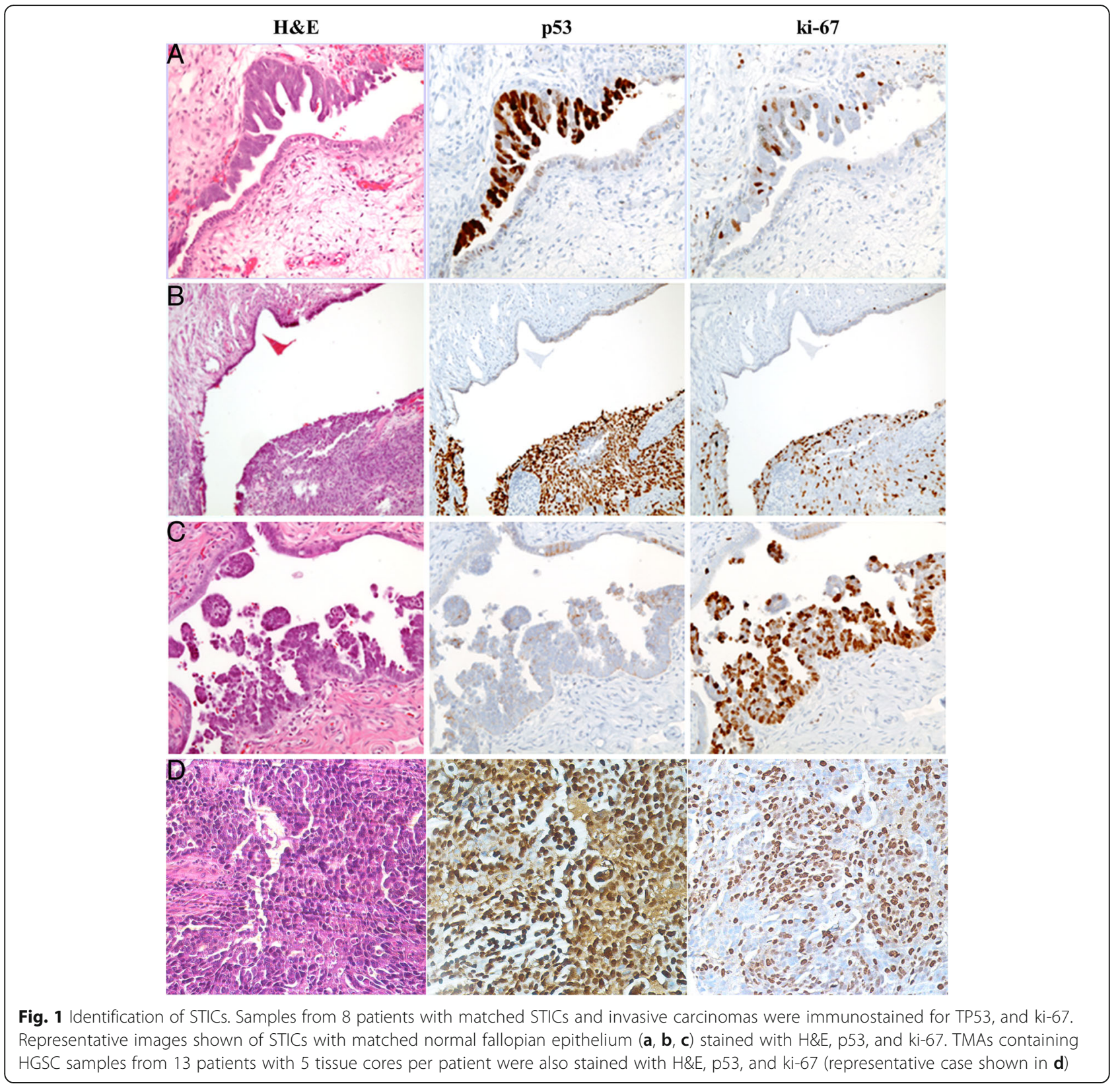

large T-antigen immortalized normal fallopian tube cell line FT2021 using a TaqMan ${ }^{\circ}$ Copy Number assay (Fig. 6a, left). Previously TCGA reported cell lines COV318 and OAW42 were amplified. Using the TaqMan ${ }^{\circ}$ Copy Number assay and determination by the CopyCaller ${ }^{\mathrm{m}}$ Software, this was confirmed. Additional cell lines such as OV2008 and PE014 were also identified by CopyCaller ${ }^{\mathrm{ru}}$ Software as amplified. A2780, Kuramochi, and the SV40 large T antigen immortalized normal fallopian tube cell line FT2021 had normal copy number of ADRM1, by this approach. However, OVCAR3 was also amplified by CopyCaller $^{\text {Tax }}$ Software, differing from TGCA results. Among these cell lines, ADRM1 mRNA levels (Fig. 6a, right) are not correlated to $A D R M 1$ amplification, although this may reflect the relatively small sample size. Further, the panel of cell lines showed largely similar RPN13 protein levels by Western blot (Fig. 6b) regardless of amplification status, suggesting possible post-transcriptional regulation of RPN13 expression.

To address the hypothesis that variability in ADRM1 amplification and mRNA expression could potentially alter sensitivity to RA190, XTT cell viability assays were run on HGSC cell lines A2780, Kuramochi, PE014, OVCAR3, OAW42, and COV318, with differing ADRM1 copy number. All cell lines were sensitive to RA190, with IC50s ranging from 1.2-2.3 $\mu \mathrm{M}$. There was no apparent 
Table 1 TMA patient information

\begin{tabular}{lllll}
\hline Patient ID & Age & Race & Stage/grade & \\
\hline Patient 1 & 47 & W & IA & HG \\
Patient 2 & 51 & W & IIIC & HG \\
Patient 3 & 61 & W & IIIC & HG \\
Patient 4 & 58 & B & IIIC & HG \\
Patient 5 & 69 & B & IIIC & HG \\
Patient 6 & 76 & W & IIIC & HG \\
Patient 7 & 61 & W & IIIC & HG \\
Patient 8 & 60 & B & IIIC & HG \\
Patient 9 & 61 & W & IV & HG \\
Patient 10 & unknown & unknown & unknown & HG \\
Patient 11 & 55 & Alaskan & IIIC & HG \\
Patient 12 & 58 & B & IIIC & HG \\
Patient 13 & 75 & W & IIIC & HG \\
\hline Age, race, a & star 13 patiens inc &
\end{tabular}

Age, race, and stage for 13 patients included in TMA analysis $W$ white, $B$ Black, HG High-grade, unk unknown

correlation between $A D R M 1$ copy number variation and sensitivity to RA190 in this limited panel of cell lines (Fig. 6c).

\section{Discussion}

The overabundance of misfolded proteins and consequent increased reliance on the ubiquitin proteasome system to relieve proteotoxic stress make ovarian carcinoma an attractive candidate for targeted proteasome inhibition. Recent inhibitors targeting the $19 \mathrm{~S}$ ubiquitin receptor RPN13 have demonstrated therapeutic potential against cultured cancer cell lines as well as in vivo models and raised questions about RPN13 expression patterns and its functional role in cancer biology [41]. In this study, we have validated a chromogenic in situ hybridization assay to visualize $A D R M 1$ mRNA levels in normal fallopian tube epithelium, STICs, and HGSCs to determine their relationship to ovarian carcinogenesis. We show that overexpression of ADRM1 mRNA is an early event found in all probed STIC, the precursor lesions of HGSC. Further, we confirmed expression of RPN13 protein in normal fallopian tube epithelium, STIC and the ovarian tumors. The specificity of $A D R M 1$ overexpression to developing tumors implies it may be reflect an early response to accommodate an overabundance of misfolded proteins found even in the cancer precursor. Additionally, the increased ADRM1 mRNA expression with respect to disease stage and recurrence further argues that RPN13 may contribute to disease progression. However, we did not see differences in expression between intraepithelial (STIC) and invasive (HGSC) carcinomas. [30]. Recent studies have shown that of the 214 known genes mapping to chromosome 20q13, overexpression of ADRM1 mRNA is the most highly correlated with this chromosomal amplification [30]. Further, in vitro overexpression of $A D R M 1$ has led to increase in proliferation and migration of transfected ovarian cancer cell line, ES2, and has been correlated to shortened survival and more rapid relapse [53]. However, the specific pathways affected by $A D R M 1$ overexpression have yet to be determined.

ADRM1 mRNA overexpression is associated with chromosome 20q13 amplification [30], but was not obviously associated with concurrent protein level changes in ovarian cancer cell lines. RPN13 protein was expressed in all normal fallopian tube epithelium, STICs, and HGSC samples tested. Patient tumor tissue samples as well as ovarian cancer cell lines show relatively consistent protein levels regardless of ADRM1 mRNA expression levels suggesting that RPN13 expression is heavily post-transcriptionally regulated.

Proteasome components exhibit coordinate regulation mediated by Nrf1 signaling in response to partial proteasome inhibition [54, 55], and we have described their upregulation in ovarian cancer in association with ubiquitin-proteasome system (UPS) stress and increased metabolic burden [56]. Taken together, this implies the upregulation of ADRM1 mRNA in STIC and HGSC may reflect their increased UPS and metabolic burden when compared to normal fallopian tube epithelium.

The consistent RPN13 protein expression in HGSC and the lack of impact of $A D R M 1$ amplification upon sensitivity to RA190 suggest that RPN13 may still be used as a therapeutic drug target without major dose adjustments for $A D R M 1$ amplification status. Indeed, all of the ovarian cancer cell lines tested were sensitive to RA190, although the panel warrants expansion. Another factor may be a second target; RA190 appears to adduct to both RPN13 and UCH37 to inhibit proteasome function synergistically [57]. RA190 or similar compounds have promise for treatment of patients whose disease becomes resistant to proteasome inhibition by other proteasome licensed inhibitors, such as bortezomib. Initial studies have shown that RA190 is active against mouse models of ovarian cancer, ES2 and ID8, although they are also sensitive to bortezomib [41].

\section{Conclusions}

We show that ADRM1 mRNA overexpression is an early event in HGSC carcinogenesis. This is associated with TP53 mutation and increased burden of misfolded proteins in carcinomas that likely renders the cancer cells particularly sensitive to RPN13 inhibitors. However, regardless of ADRM1 amplification status the RPN13 protein levels are relatively consistent across ovarian cancer cell lines. Since ADRM1 amplification was also not correlated with sensitivity to RA190, this suggests 


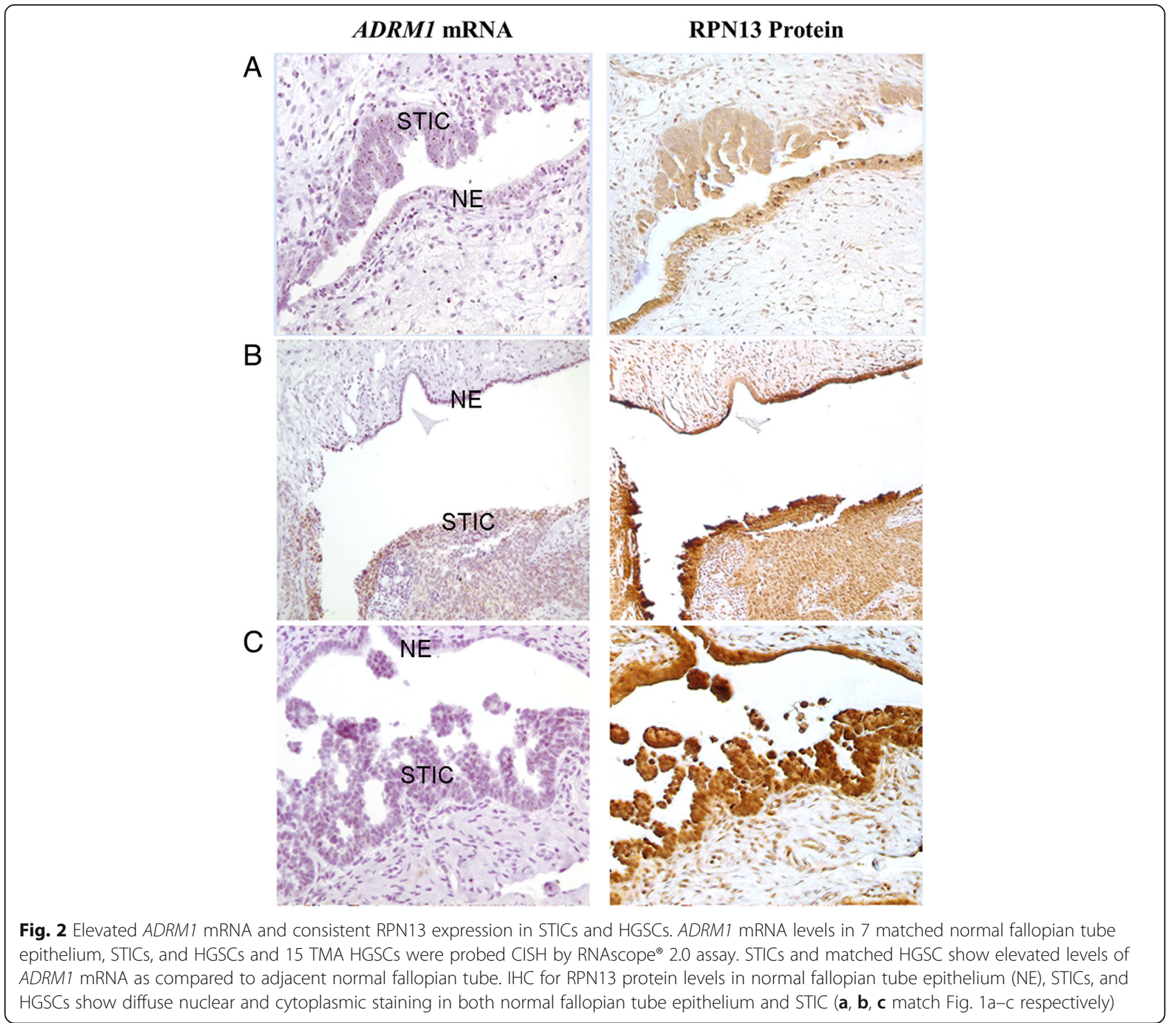

it can be tested against ovarian cancer regardless of increased ADRM1 copy number.

\section{Methods}

\section{Tissue specimens and cell culture}

Studies on archived formalin-fixed, paraffin-embedded ovarian carcinoma tissue specimens selected from cases in the Department of Pathology at The Johns Hopkins Hospital were approved by the Johns Hopkins University Institutional Review Board. A total of 20 specimens were selected with pathological diagnoses of high grade serous carcinoma, of which 7 also exhibited STIC. One pathologist (DX) reviewed all of the slides for all cases independently to assess the staining, and STIC diagnoses were confirmed by 3 pathologists (JDS, AVY, DX). OAW42, OV2008, COV318 cells were maintained in Dulbecco's modified Eagle medium (DMEM) with 10\% fetal bovine serum, $100 \mathrm{U}$ penicillin and streptomycin, $1 \mathrm{U}$ non-essential amino acids, $1 \mathrm{mM}$ sodium pyruvate. A2780, Kuramochi, OVCAR3 cell lines were maintained in RPMI medium supplemented with $10 \%$ fetal bovine serum, $100 \mathrm{U}$ penicillin and streptomycin, $1 \mathrm{U}$ nonessential amino acids, $1 \mathrm{mM}$ sodium pyruvate (Gibco, Life Technologies, Grand Island NY).

\section{Western blots}

Prior to lysate preparation, adherent cells were released with $0.05 \%$ Trypsin-EDTA (Gibco, Life Technologies, Grand Island NY), then harvested by centrifugation at $2500 \times \mathrm{g}$ for $4 \mathrm{~min}$. The cell pellets were washed $3 \times$ with $1 \mathrm{~mL}$ DPBS followed by centrifugation at $2500 \times \mathrm{g}$ for $4 \mathrm{~min}$. Pellets were lysed using $1 \mathrm{~mL}$ of M-PER ${ }^{\circ}$ Mammalian Protein Extraction Reagent for $\sim 100 \mu \mathrm{L}$ of cells and processed as per manufacturer's instructions (Pierce, 


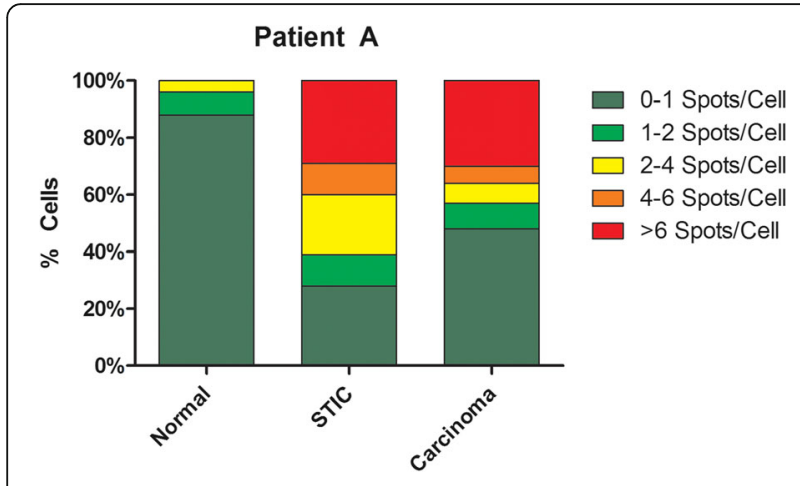

Fig. 3 SpotStudio Quantification of RNA-CISH in fallopian tube epithelium, STICs, and HGSCs. Using ACD SpotStudio Software, single cell analysis for CISH were done on all 7 matched normal, STIC, and HGSC samples. STICS and HGSC showed a higher percentage of cells overexpressing ADRM1 mRNA as compared to fallopian tube epithelium. Distribution of cells containing higher number of ADRM1 mRNA spots increases FT progresses to STIC and carcinoma. Collectively, STIC and HGSC exhibited significantly different levels as compared to normal ( $P=0.009, P=0.003$, paired T-test). One representative case (Patient $A$ ) is presented, and the remainder (Patients B-G) are presented in Additional file 4: Figure S4

ThermoFisher Scientific, Rockford IL). The protein concentration of cell lysates was quantified using a BCA Assay Kit (Pierce, ThermoFisher Scientific, Rockford IL). Intensity of RPN13 and actin bands was analyzed using the NCI software ImageJ (http://rsb.info.nih.gov/ij/index.html) and their ratio determined for normalization.

\section{Cell viability assay}

Adherent cell lines were seeded at 2000 cells/well in $100 \mu \mathrm{L}$ of culture medium in 96-well plates. Following overnight incubation, wells were treated with serially diluted drug at specified concentrations in $100 \mu \mathrm{L}$ and incubated for $48 \mathrm{~h}$. Cells were incubated for the final $2 \mathrm{~h}$ at $37{ }^{\circ} \mathrm{C}$ with $20 \mu \mathrm{L}$ of $5 \mathrm{mg} / \mathrm{mL}$ tetrazolium salt XTT (Sigma-Aldrich, St. Louis MO). To visualize, plates were lightly tapped against paper towels, crystals were dissolved in $100 \mu \mathrm{L}$ of DMSO (Sigma-Aldrich, St. Louis $\mathrm{MO}$ ), and absorbance of each well read at $490 \mathrm{~nm}$ using a Benchmark Plus microplate spectrophotometer (BIO-RAD, Hercules CA).

\section{TaqMan $^{\circ}$ qRT-PCR and copy number variation assay}

Prior to lysate preparation, adherent cells were treated to $0.05 \%$ Trypsin-EDTA then centrifuged at $2500 \times \mathrm{g}$ for $4 \mathrm{~min}$. Remaining cell pellets were washed $3 \times$ by $1 \mathrm{ml}$ DPBS followed by centrifugation at $2500 \times \mathrm{g}$ for $4 \mathrm{~min}$. For qRT-PCR, RNA was harvested using an RNeasy Mini Kit (Qiagen Sciences, Germantown MD) followed by reverse transcription by iScript Reverse Transcription Supermix (BIO-RAD, Hercules CA) per the manufacturer's instructions. qPCR was performed on a Bio-Rad
iCycler iQ ${ }^{\mathrm{m}}$ Real-Time PCR Detection System using TaqMan Fast Advanced Master Mix and ADRM1 TaqMan probes (Cat. \# 4351372) (Applied Biosystems, ThermoFisher Scientific, Rockford IL). Fold-change was calculated using the Livak method and normalized to reference gene GAPDH.

For the Copy Number Variation Assay, gDNA was harvested using a DNeasy Blood \& Tissue Kit (Qiagen Sciences, Germantown MD). DNA was quantified using UV absorbance determined with a Nanodrop ${ }^{\mathrm{m}}$. The realtime-PCR assay was run according Applied Biosystems' TaqMan Copy Number protocol using $20 \mathrm{ng}$ of DNA, TaqMan Genotyping Master Mix, and ADRM1 TaqMan Copy Number Probe (Cat. \# 4400291) and was quantified using CopyCaller ${ }^{\text {Tw }}$ Software.

\section{Chromogenic in situ hybridization}

Custom RNA in situ hybridization probes (Advanced Cell Diagnostics, Inc.) were prepared to detect the fulllength $A D R M 1$ mRNA. RNAscope ${ }^{\circ}$ assays were performed using the RNAscope ${ }^{\circ}$ 2.0 FFPE Brown Reagent kit. Glass-mounted $4 \mu \mathrm{m}$ sections of formalin-fixed, paraffin-embedded tissue (FFPE) were baked overnight at $65{ }^{\circ} \mathrm{C}$, then deparaffinized in three $10 \mathrm{~min}$ incubations in xylene, with the last incubation in fresh xylene. Endogenous peroxidase activity was blocked for $10 \mathrm{~min}$ (P1) followed by antigen retrieval by both heat (P2) and protease (P3). Protease antigen retrieval (P3) was diluted 1:5 in sterile DPBS (Gibco, Life Technologies, Grand Island NY) and incubated for $30 \mathrm{~min}$. ADRM1 probe hybridization and amplifications were completed according to manufacturer's instructions. After washing, an HRP-based amplification system was then used to detect the target probes by $10 \mathrm{~min}$ incubation with manufacturer supplied DAB solutions. To ensure RNA integrity and assay procedure, adjacent sections were also hybridized with a probe to the endogenous housekeeping protein peptidyl prolyl isomerase B (PPIB). To control for non-specific staining, control probe against bacterial protein dihydrodipicolinate reductase (dapB) was used on adjacent slides. Quantification of slides was done using ACD supplied SpotStudio Software.

\section{ADRM1 knockout cell line}

Stable ADRM1 knockout cell line was established using CRISPR/Cas9-mediated gene targeting strategy in the HCT116 Cell line. A 0.9-kb fragment upstream of exon 4 as a $5^{\prime}$-homologous arm and a 1.0-kb fragment downstream of exon 4 as a $3^{\prime}$-homologous arm in ADRM1 gene (encoding RPN13) was amplified by PCR using genomic DNA from HCT116 cells as a template. The primers used were $5^{\prime}$-AACTCGAGTGAAGGGGACCA CCGTGACTCCG-3' and 5' -TTGAATTCTTGGACCC TGCCTTGAACTTCAGC-3' for the 0.9-kb fragment, 


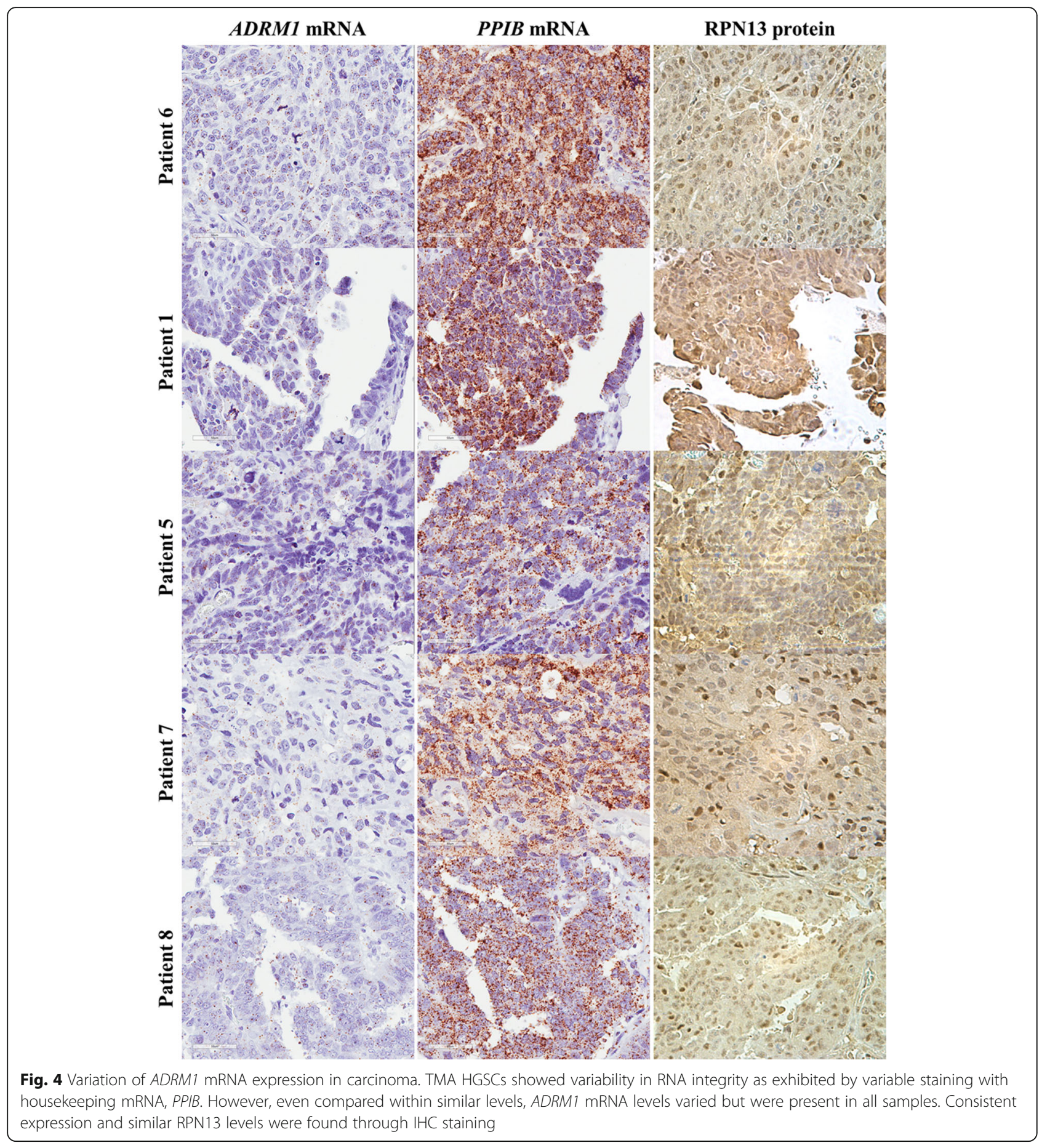

and $5^{\prime}$-AAGGATCCATGCTGGCCCTGGTTCTAACG ATG-3' and 5' ${ }^{\prime}$-TTTGCGGCCGCTCCGAAGGCACTT AGCTGCTGC-3' for the 1.0-kb fragment. A targeting vector was constructed by sequentially subcloning the $5^{\prime}$-arm, the puromycin resistance gene cassette, and the $3^{\prime}$-arm into pBluescript II in order to delete exon 4 of $A D R M 1$ gene. sgRNA-encoding DNA oligos, 5' AAACTGCGATTCGGTTAGGAACTTC $-3^{\prime}$ and $5^{\prime}$ -
CACCGAAGTTCCTAACCGAATCGCA -3', target 39-bp downstream and of exon 4. The pair of annealed oligos was subcloned into the BbsI site of pX330 (Addgene 42,230), which expresses sgRNA and Cas9 simultaneously. The targeting vector and the pX330 plasmid encoding each sgRNA were transfected into HCT116 cells. The cells were cultured in DMEM supplemented with $10 \%$ FBS and $4 \mu \mathrm{g} / \mathrm{ml}$ puromycin for 10 days. Colonies were then picked up, and 


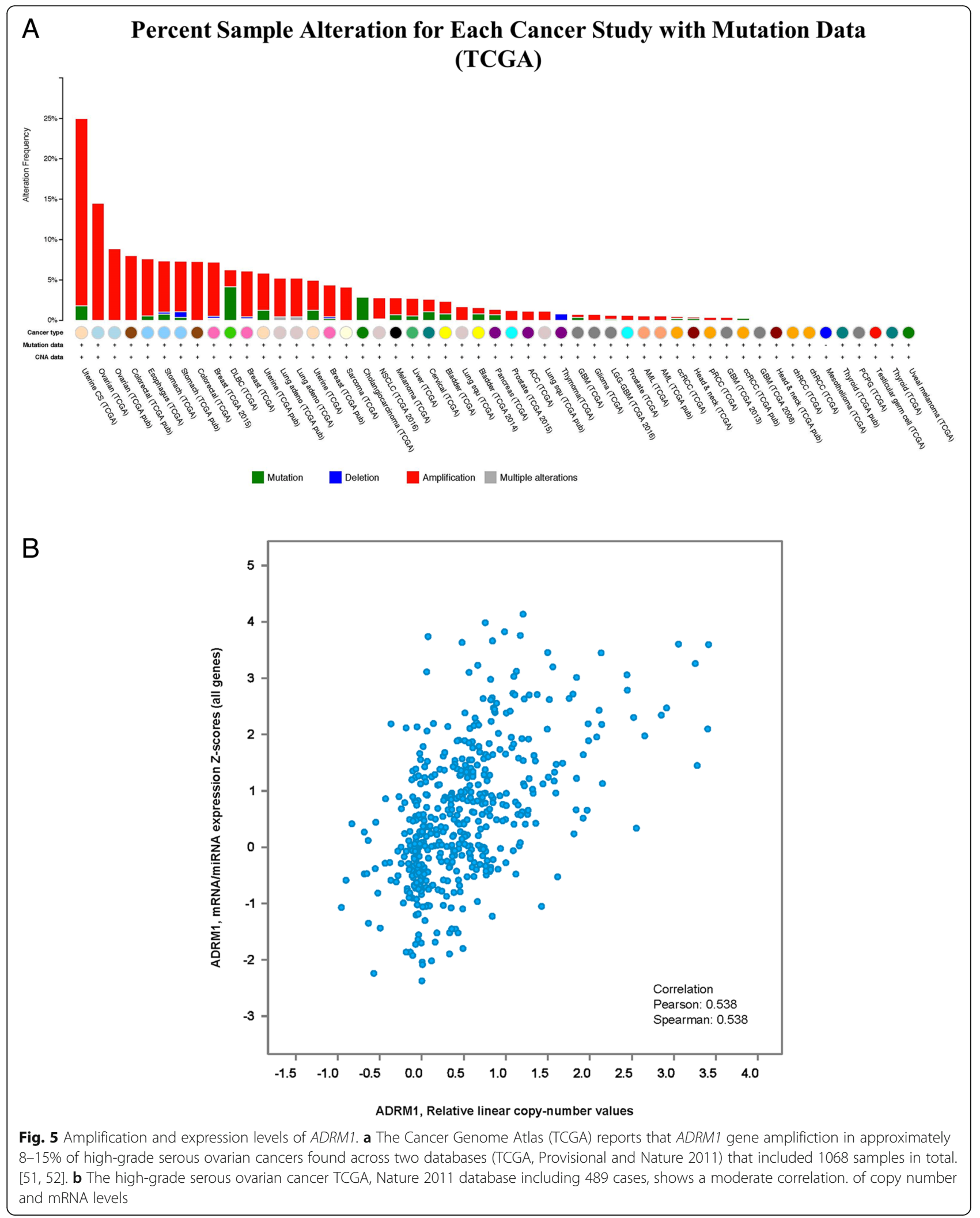




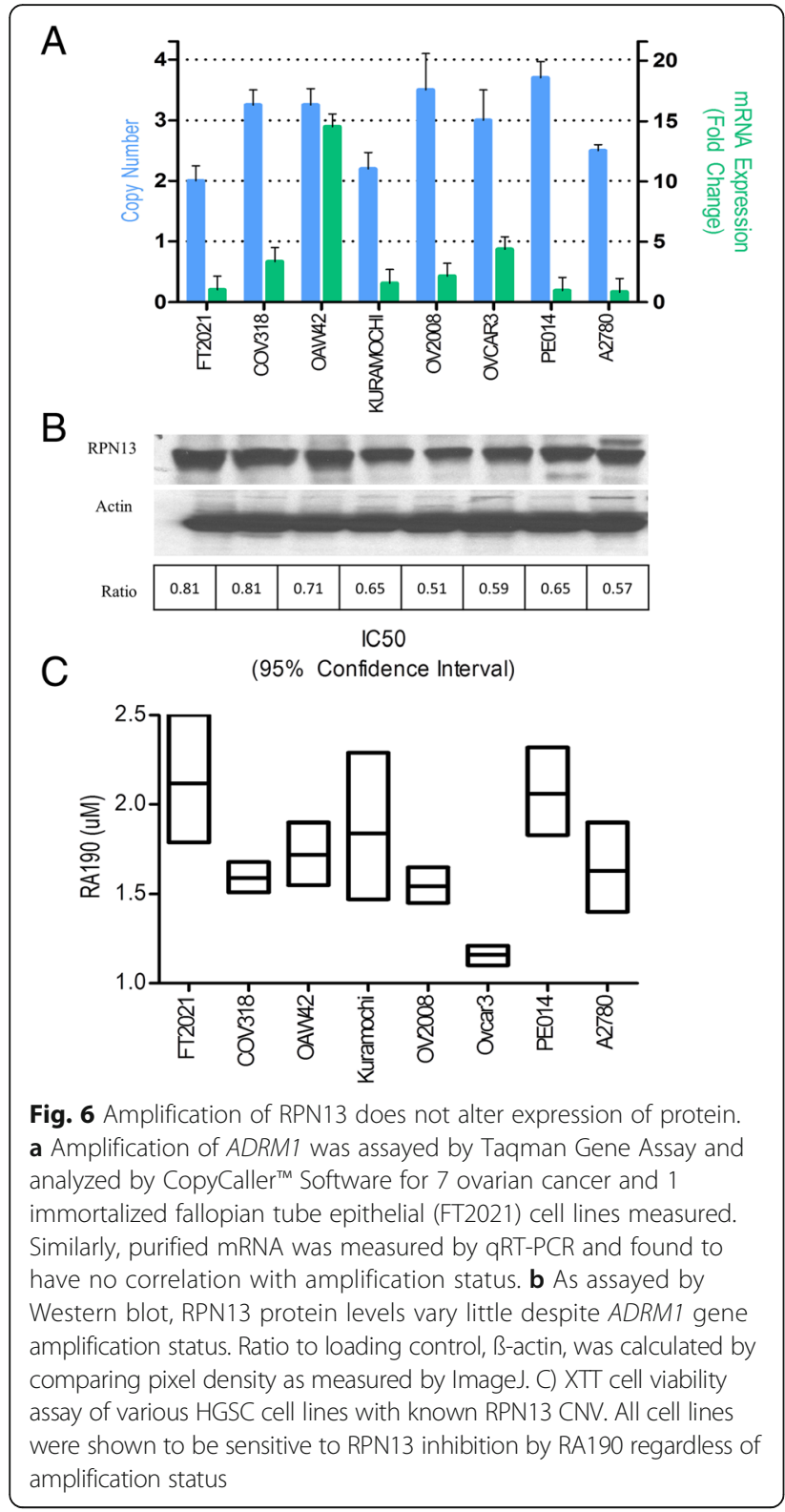

deficiency of Rpn13 proteins was screened by immunoblot analysis of cell lysates.

\section{Immunohistochemistry}

RPN13 was stained by following PowerVision Poly-HRP IHC Detection system protocol (Leica Biosystem). FFPE sections were deparaffinized in xylene, followed by rehydration in graded ethanol. Antigen retrieval was performed by steaming specimens at $100{ }^{\circ} \mathrm{C}$ for $20 \mathrm{~min}$ in Target Retrieval Solution (Dako) and subsequently washed in Tris-buffered saline with Tween 20 (TBST, 0.05\% Tween 20). Endogenous peroxidase was blocked, by treatment of slides with Dual Endogenous EnzymeBlocking Reagent (Dako) for $5 \mathrm{~min}$ at room temperature. Sections were covered with 1:500 dilution of mouse monoclonal ADRM1 Antibody (F-12) supplied by Santa Cruz Biotechnology (sc-166,754) diluted with Antibody Dilution Buffer (ChemMate) and then incubated at room temperature for $45 \mathrm{~min}$. Slides were then washed with TBST, followed by incubation with PowerVision PolyHRP Anti-Mouse IgG for $30 \mathrm{~min}$ at room temperature. After three washes in TBST, sections were treated with DAB chromogen (3, 3 '-diaminobenzidine tetrahydrochloride; Sigma) for $20 \mathrm{~min}$ in the dark. Sections were counterstained with Mayer's hematoxylin (Dako), dehydrated with ethanol and xylene, and mounted permanently.

\section{Additional files}

Additional file 1: Figure S1. Validation of a highly sensitive and specific ADRM1 Chromogenic In Situ Hybridization (CISH) assay. A) ADRM1 mRNA levels in samples from 4 HGSC patients with matched FFPE blocks and frozen tissue were compared in parallel by RNAscope ${ }^{\oplus} 2.0$ assay and qRT-PCR respectively. Increase in mRNA by qRT-PCT was mirrored in RNAscope $^{\oplus} 2.0$ assay validating the quantitative capabilities of the $\mathrm{CISH}$ assay. Images taken at 40x magnification. B) Protein lysates of same patients were probed by Western blot and found to express similar levels of RPN13 protein despite variable ADRM1 mRNA levels. Ratio of RPN13 to loading control, ß-actin, were calculated by comparing pixel density as measured by ImageJ. (TIFF $1689 \mathrm{~kb}$ )

Additional file 2: Figure S2. PPIB positive control pictures for STIC. Matched FT, STIC, and HGSC samples as well as TMA HGSCs were probed for housekeeping mRNA, PPIB, by RNA-CISH to assess RNA integrity. (TIFF $9327 \mathrm{~kb}$ )

Additional file 3: Figure S3. SpotStudio Quantification of RNA-CISH. Using ACD SpotStudio Software, single cell analysis for CISH were done on all 7 matched normal, STIC, and HGSC samples. Example image shown. (TIFF $3493 \mathrm{~kb}$ )

Additional file 4: Figure S4. Distribution of $A D R M 1 \mathrm{mRNA}$ expression per cell in matched FT, STIC, and HGSC samples. RNA-CISH probed samples were analyzed using ACD SpotStudio for estimated ADRMT mRNA spots per cell. Distribution of high ADRM1 expression cells are highest in STIC and HGSC regions of interest in all cases. (TIFF $1160 \mathrm{~kb}$ )

Additional file 5: Figure S5. Verification of anti-RPN13 antibody specificity. ADRM1 knockout HCT116 cell line and its parental line were probed for RPN13 expression by both $\mathrm{HC}$ and Western blot. No staining in knockout cells were seen by either assay. (TIFF 3640 kb)

Additional file 6: Figure S6. Consistent RPN13 protein expression in TMA HGSC (Part 1). TMA HGSC samples were assessed for RPN13 mRNA and protein expression. mRNA levels varied between samples however, protein levels remained similar between samples. (TIFF 6401 kb)

Additional file 7: Figure S7. Consistent RPN13 protein expression in TMA HGSC (Part 2). This shows additional cases, stained as described in Figure S6. (TIFF $9590 \mathrm{~kb}$ )

\section{Abbreviations}

CISH: Chromogenic in situ hybridization; CP: 20 S Core particle;

DAB: 3, 3'-diaminobenzidine tetrahydrochloride; FFPE: Formalin-fixed, paraffin embedded; HGSC: High grade serous carcinoma; MM: Multiple myeloma; PPIB: Peptidyl prolyl isomerase B; RP : 195 regulatory particle; STIC: Serous tubal intraepithelial carcinoma; TCGA: The cancer genome atlas; UPR: Unfolded protein response; UPS: Ubiquitin-proteasome system; XTT: 2,3-Bis-(2-Methoxy-4-Nitro-5-Sulfophenyl)-2H-Tetrazolium-5-Carboxanilide

\section{Acknowledgements}

We thank Karen Sfanos and Jessica Hicks for guidance in optimizing the immunohistochemistry protocols. 


\section{Funding}

Grant support was provided by National Institutes of Health grant P50 CA098252, the Alleghany Health Network-Johns Hopkins Cancer Research Fund, and the Ovarian Cancer Research Fund Alliance \#458972 (to RKA, TLW. and RBSR.).

\section{Availability of data and materials}

The datasets used and/or analyzed during the current study are available from the corresponding author on reasonable request.

\section{Authors' contributions}

RTJ, AVY, and RBSR conceived of and designed the experiments. JH and SM developed knockout cell line for studies. Drug assays were done by RTJ and RKA. AVY, JDS, and DX provided tissue samples and pathology guidance. RTJ and RBSR wrote the manuscript with revisions from all authors. All authors read and approved the final manuscript.

\section{Authors' information}

Dr. Yemelyanova's current affiliation is Department of Pathology, University of Texas MD Anderson Cancer Center, Houston, TX. The opinions and assertions herein are the private views of the authors and do not purport to be official or to reflect the position of the US FDA or any other part of the US Government. This work was not prepared as part of Dr. Seidman's official government duties.

\section{Ethics approval and consent to participate}

The studies herein under waiver of consent were approved by the Johns Hopkins University Institutional Review Board.

\section{Consent for publication}

Not applicable.

\section{Competing interests}

Under a licensing agreement between Pontifax/PI Therapeutics and the Johns Hopkins University, Drs. Anchoori and Roden are entitled to royalties on an invention (RA190) described in this article. This arrangement has been reviewed and approved by the Johns Hopkins University in accordance with its competing interests policies.

\section{Publisher's Note}

Springer Nature remains neutral with regard to jurisdictional claims in published maps and institutional affiliations.

\section{Author details}

'Department of Pathology, The Johns Hopkins University, Baltimore, MD 21231, USA. ${ }^{2}$ Department of Oncology, The Johns Hopkins University, Baltimore, MD 21231, USA. ${ }^{3}$ Departments of Gynecology and Obstetrics, The Johns Hopkins University, Baltimore, MD 21231, USA. ${ }^{4}$ Division of Gynecologic Pathology, The Johns Hopkins University, Baltimore, MD 21231, USA. ${ }^{5}$ Laboratory of Protein Metabolism, The University of Tokyo, Bunkyo-ku, Tokyo, Japan. ${ }^{6}$ Division of Molecular Genetics and Pathology, Center for Devices and Radiological Health, Food and Drug Administration, Silver Spring, MD 20993, USA.

Received: 25 April 2017 Accepted: 18 July 2017

Published online: 07 August 2017

\section{References}

1. Kuhn E, Wu RC, Guan B, Wu G, Zhang J, Wang Y, Song L, Yuan X, Wei L, Roden $\mathrm{RB}$, et al. Identification of molecular pathway aberrations in uterine serous carcinoma by genome-wide analyses. J Natl Cancer Inst. 2012; 104(19):1503-13.

2. Cancer Genome Atlas Research Network. Integrated genomic analyses of ovarian carcinoma. Nature. 2011;474(7353):609-15.

3. Cho KR, Shih le M. Ovarian cancer. Annu Rev Pathol. 2009;4:287-313.

4. Cordenonsi M, Dupont S, Maretto S, Insinga A, Imbriano C, Piccolo S. Links between Tumor Suppressors: p53 Is Required for TGF- $\beta$ Gene Responses by Cooperating with Smads. Cell. 2003;113(3):301-14.

5. Bast RC, Hennessy B, Mills GB. The biology of ovarian cancer: new opportunities for translation. Nat Rev Cancer. 2009;9(6):415-28.
6. Petitjean A, Mathe E, Kato S, Ishioka C, Tavtigian SV, Hainaut P, Olivier M. Impact of mutant p53 functional properties on TP53 mutation patterns and tumor phenotype: lessons from recent developments in the IARC TP53 database. Hum Mutat. 2007:28(6):622-9.

7. Mittal N, Srinivasan R, Gupta N, Rajwanshi A, Nijhawan R, Gautam U, Sood S, Dhaliwal L. Secretory cell outgrowths, p53 signatures, and serous tubal intraepithelial carcinoma in the fallopian tubes of patients with sporadic pelvic serous carcinoma. Indian J Pathol Microbiol. 2016;59(4):481-8.

8. Verhaak RG, Tamayo P, Yang JY, Hubbard D, Zhang H, Creighton CJ, Fereday S, Lawrence M, Carter SL, Mermel CH, et al. Prognostically relevant gene signatures of high-grade serous ovarian carcinoma. J Clin Invest. 2013;123(1):517-25.

9. Horn LC, Kafkova S, Leonhardt K, Kellner C, Einenkel J. Serous tubal in situ carcinoma (STIC) in primary peritoneal serous carcinomas. Int J Gynecol Pathol. 2013;32(4):339-44.

10. Kuhn E, Kurman RJ, Vang R, Sehdev AS, Han G, Soslow R, Wang TL, Shih IM. TP53 mutations in serous tubal intraepithelial carcinoma and concurrent pelvic high-grade serous carcinoma-evidence supporting the clonal relationship of the two lesions. J Pathol. 2012;226(3):421-6.

11. Walerych D, Lisek K, Sommaggio R, Piazza S, Ciani Y, Dalla E, Rajkowska K, Gaweda-Walerych K, Ingallina E, Tonelli C, et al. Proteasome machinery is instrumental in a common gain-of-function program of the p53 missense mutants in cancer. Nat Cell Biol. 2016;18(8):897-909.

12. Bazzaro M, Lee MK, Zoso A, Stirling WL, Santillan A, Shih le M, Roden RB. Ubiquitin-proteasome system stress sensitizes ovarian cancer to proteasome inhibitor-induced apoptosis. Cancer Res. 2006;66(7):3754-63.

13. Saulle E, Petronelli A, Pasquini L, Petrucci E, Mariani G, Biffoni M, Ferretti G, Scambia G, Benedetti-Panici P, Cognetti F, et al. Proteasome inhibitors sensitize ovarian cancer cells to TRAIL induced apoptosis. Apoptosis. 2007;12(4):635-55.

14. Heubner M, Wimberger P, Dahlmann B, Kasimir-Bauer S, Kimmig R, Peters J, Wohlschlaeger J, Sixt SU. The prognostic impact of circulating proteasome concentrations in patients with epithelial ovarian cancer. Gynecol Oncol. 2011:120(2):233-8

15. Bedford L, Lowe J, Dick LR, Mayer RJ, Brownell JE. Ubiquitin-like protein conjugation and the ubiquitin-proteasome system as drug targets. Nat Rev Drug Discov. 2011;10(1):29-46.

16. Chen S, Blank JL, Peters T, Liu XJ, Rappoli DM, Pickard MD, Menon S, Yu J, Driscoll DL, Lingaraj T, et al. Genome-wide siRNA screen for modulators of cell death induced by proteasome inhibitor bortezomib. Cancer Res. 2010;70(11):4318-26

17. Aghajanian C, Blessing JA, Darcy KM, Reid G, DeGeest K, Rubin SC, Mannel RS, Rotmensch J, Schilder RJ, Riordan W. A phase II evaluation of bortezomib in the treatment of recurrent platinum-sensitive ovarian or primary peritoneal cancer: a Gynecologic Oncology Group study. Gynecol Oncol. 2009;115(2):215-20.

18. Aghajanian C, Dizon DS, Sabbatini P, Raizer JJ, Dupont J, Spriggs DR. Phase I trial of bortezomib and carboplatin in recurrent ovarian or primary peritoneal cancer. J Clinl Oncol. 2005;23(25):5943-9.

19. Aghajanian C, Soignet S, Dizon DS, Pien CS, Adams J, Elliott PJ, Sabbatini P, Miller V, Hensley ML, Pezzulli S, et al. A phase I trial of the novel proteasome inhibitor PS341 in advanced solid tumor malignancies. Clin Cancer Res. 2002;8(8):2505-11.

20. Zhao Y, Foster NR, Meyers JP, Thomas SP, Northfelt DW, Rowland KM Jr, Mattar BI, Johnson DB, Molina JR, Mandrekar SJ, et al. A phase I/II study of bortezomib in combination with paclitaxel, carboplatin, and concurrent thoracic radiation therapy for non-small-cell lung cancer: North Central Cancer Treatment Group (NCCTG)-N0321. J Thorac Oncol. 2015;10(1):172-80.

21. Parma G, Mancari R, Del Conte G, Scambia G, Gadducci A, Hess D, Katsaros D, Sessa C, Rinaldi A, Bertoni F, et al. An open-label phase 2 study of twice-weekly bortezomib and intermittent pegylated liposomal doxorubicin in patients with ovarian cancer failing platinum-containing regimens. Int J Gynecol Cancer. 2012;22(5):792-800.

22. Falchook GS, Duvic M, Hong DS, Wheler J, Naing A, Lim J, Kurzrock R. Age-stratified phase I trial of a combination of bortezomib, gemcitabine, and liposomal doxorubicin in patients with advanced malignancies. Cancer Chemother Pharmacol. 2012;69(5):1117-26.

23. Kobrinsky B, Joseph SO, Muggia F, Liebes L, Beric A, Malankar A, Ivy P, Hochster H. A phase I and pharmacokinetic study of oxaliplatin and bortezomib: activity, but dose-limiting neurotoxicity. Cancer Chemother Pharmacol. 2013;72(5):1073-8. 
24. Chen X, Lee BH, Finley D, Walters KJ. Structure of proteasome ubiquitin receptor hRpn13 and its activation by the scaffolding protein hRpn2. Mol Cell. 2010;38(3):404-15.

25. Hamazaki J, lemura S, Natsume T, Yashiroda H, Tanaka K, Murata S. A nove proteasome interacting protein recruits the deubiquitinating enzyme UCH37 to 265 proteasomes. EMBO J. 2006;25(19):4524-36.

26. Qiu XB, Ouyang SY, Li CJ, Miao S, Wang L, Goldberg AL. hRpn13/ADRM1/ GP110 is a novel proteasome subunit that binds the deubiquitinating enzyme, UCH37. EMBO J. 2006;25(24):5742-53.

27. Yao T, Song L, Xu W, DeMartino GN, Florens L, Swanson SK, Washburn MP, Conaway RC, Conaway JW, Cohen RE. Proteasome recruitment and activation of the Uch37 deubiquitinating enzyme by Adrm1. Nat Cell Biol. 2006;8(9):994-1002.

28. Simins AB, Weighardt $H$, Weidner KM, Weidle UH, Holzmann B. Functional cloning of ARM-1, an adhesion-regulating molecule upregulated in metastatic tumor cells. Clin Exp Metastasis. 1999;17(8):641-8.

29. Pilarsky C, Wenzig M, Specht T, Saeger HD, Grutzmann R. Identification and validation of commonly overexpressed genes in solid tumors by comparison of microarray data. Neoplasia. 2004;6(6):744-50.

30. Fejzo MS, Dering J, Ginther C, Anderson L, Ramos L, Walsh C, Karlan B, Slamon DJ. Comprehensive analysis of $20 \mathrm{q} 13$ genes in ovarian cancer identifies ADRM1 as amplification target. Genes Chromosomes Cancer. 2008;47(10):873-83.

31. Fejzo MS, Ginther C, Dering J, Anderson L, Venkatesan N, Konecny G, Karlan B, Slamon DJ. Knockdown of ovarian cancer amplification target ADRM1 leads to downregulation of GIPC1 and upregulation of RECK. Genes Chromosomes Cancer. 2011;50(6):434-41.

32. Fejzo MS, Anderson L, von Euw EM, Kalous O, Avliyakulov NK, Haykinson MJ, Konecny GE, Finn RS, Slamon DJ. Amplification target ADRM1: role as an oncogene and therapeutic target for ovarian cancer. Int J Mol Sci. 2013; 14(2):3094-109.

33. Carvalho B, Postma C, Mongera S, Hopmans E, Diskin S, van de Wiel MA van Criekinge W, Thas O, Matthai A, Cuesta MA, et al. Multiple putative oncogenes at the chromosome $20 \mathrm{q}$ amplicon contribute to colorectal adenoma to carcinoma progression. Gut. 2009;58(1):79-89.

34. Chen W, Hu XT, Shi QL, Zhang FB, He C. Knockdown of the novel proteasome subunit Adrm1 located on the 20q13 amplicon inhibits colorectal cancer cell migration, survival and tumorigenicity. Oncol Rep. 2009;21(2):531-7.

35. Jang SH, Park JW, Kim HR, Seong JK, Kim HK. ADRM1 gene amplification is a candidate driver for metastatic gastric cancers. Clin Exp Metastasis. 2014;31(6):727-33.

36. Fejzo MS, Anderson L, Chen HW, Anghel A, Zhuo J, Anchoori R, Roden R, Slamon DJ. ADRM1-amplified metastasis gene in gastric cancer. Genes Chromosom Cancer. 2015. doi:10.1002/gcc.22262.

37. Elangovan M, Oh C, Sukumaran L, Wojcik C, Yoo YJ. Functional differences between two major ubiquitin receptors in the proteasome; S5a and hRpn13. Biochem Biophys Res Commun. 2010;396(2):425-8.

38. Yang X, Miao X, Wen Y, Hu J, Dai W, Yin B. A possible connection between adhesion regulating molecule 1 overexpression and nuclear factor kappa B activity in hepatocarcinogenesis. Oncol Rep. 2012;28(1):283-90.

39. Zheng X, Guo Y, Chen Y, Chen M, Lin Z, Wu Y, Chen Y. Knockdown of adhesion-regulating molecule 1 inhibits proliferation in HL60 Cells. Acta Haematol. 2015;134(2):88-100.

40. Randles L, Anchoori RK, Roden RB, Walters KJ. The proteasome ubiquitin receptor hRpn13 and its interacting deubiquitinating enzyme Uch37 are required for proper cell cycle progression. J Biol Chem. 2016;291(16):8773-83.

41. Anchoori RK, Karanam B, Peng S, Wang JW, Jiang R, Tanno T, Orlowski RZ, Matsui W, Zhao M, Rudek MA, et al. A bis-benzylidine piperidone targeting proteasome ubiquitin receptor RPN13/ADRM1 as a therapy for cancer. Cancer Cell. 2013;24(6):791-805.

42. Song Y, Ray A, Li S, Das DS, Carrasco RD, Chauhan D, Anderson KC. Targeting proteasome ubiquitin receptor Rpn13 in multiple myeloma. Leukemia. 2016;30(9):1877-86.

43. Trader DJ, Simanski S, Kodadek T. A reversible and highly selective inhibitor of the proteasomal ubiquitin receptor rpn13 is toxic to multiple myeloma cells. J Am Chem Soc. 2015;137(19):6312-9.

44. Lu X, Liu F, Durham SE, Tarasov SG, Walters KJ. A high affinity hRpn2derived peptide that displaces human Rpn13 from proteasome in 293T cells. PLoS One. 2015;10(10):e0140518.

45. Ardighieri L, Mori L, Conzadori S, Bugatti M, Falchetti M, Donzelli CM, Ravaggi A, Odicino FE, Facchetti F. Identical TP53 mutations in pelvic carcinosarcomas and associated serous tubal intraepithelial carcinomas provide evidence of their clonal relationship. Virchows Archiv. 2016;469(1):61-9.

46. Kurman RJ. Origin and molecular pathogenesis of ovarian high-grade serous carcinoma. Ann Oncol. 2013:24(Suppl 10):x16-21.

47. Lim D, Oliva E. Precursors and pathogenesis of ovarian carcinoma. Pathology. 2013:45(3):229-42.

48. Visvanathan K, Vang R, Shaw P, Gross A, Soslow R, Parkash V, Shih le M, Kurman RJ. Diagnosis of serous tubal intraepithelial carcinoma based on morphologic and immunohistochemical features: a reproducibility study. Am J Surg Pathol. 2011;35(12):1766-75.

49. Vang R, Shih le M, Kurman RJ. Fallopian tube precursors of ovarian low- and high-grade serous neoplasms. Histopathology. 2013;62(1):44-58.

50. Lee Y, Miron A, Drapkin R, Nucci MR, Medeiros F, Saleemuddin A, Garber J, Birch C, Mou H, Gordon RW, et al. A candidate precursor to serous carcinoma that originates in the distal fallopian tube. J Pathol. 2007;211(1): 26-35.

51. Gao J, Aksoy BA, Dogrusoz U, Dresdner G, Gross B, Sumer SO, Sun Y, Jacobsen A, Sinha R, Larsson E, et al. Integrative analysis of complex cancer genomics and clinical profiles using the cBioPortal. Sci Signal. 2013;6(269):11.

52. Cerami E, Gao J, Dogrusoz U, Gross BE, Sumer SO, Aksoy BA, Jacobsen A, Byrne CJ, Heuer ML, Larsson E, et al. The cBio cancer genomics portal: an open platform for exploring multidimensional cancer genomics data. Cancer Discovery. 2012;2(5):401-4.

53. Soong RS, Anchoori RK, Yang B, Yang A, Tseng SH, He L, Tsai YC, Roden RB, Hung CF. RPN13/ADRM1 inhibitor reverses immunosuppression by myeloidderived suppressor cells. Oncotarget. 2016:7(42):68489-502.

54. Radhakrishnan SK, Lee CS, Young P, Beskow A, Chan JY, Deshaies RJ. Transcription factor Nrf1 mediates the proteasome recovery pathway after proteasome inhibition in mammalian cells. Mol Cell. 2010;38(1):17-28.

55. Sha Z, Goldberg AL. Proteasome-mediated processing of Nrf1 is essential for coordinate induction of all proteasome subunits and p97. Curr Biol. 2014;24(14):1573-83.

56. Bazzaro M, Lin Z, Santillan A, Lee MK, Wang MC, Chan KC, Bristow RE, Mazitschek R, Bradner J, Roden RB. Ubiquitin proteasome system stress underlies synergistic killing of ovarian cancer cells by bortezomib and a novel HDAC6 inhibitor. Clin Cancer Res. 2008;14(22):7340-7.

57. Lu X, Nowicka U, Sridharan V, Liu F, Randles L, Hymel D, Dyba M, Tarasov SG, Tarasova NI, Zhao XZ, et al. Structure of the Rpn13-Rpn2 complex provides insights for Rpn13 and Uch37 as anticancer targets. Nat Commun. 2017;8:15540.

\section{Submit your next manuscript to BioMed Central and we will help you at every step:}

- We accept pre-submission inquiries

- Our selector tool helps you to find the most relevant journal

- We provide round the clock customer support

- Convenient online submission

- Thorough peer review

- Inclusion in PubMed and all major indexing services

- Maximum visibility for your research

Submit your manuscript at www.biomedcentral.com/submit
) Biomed Central 